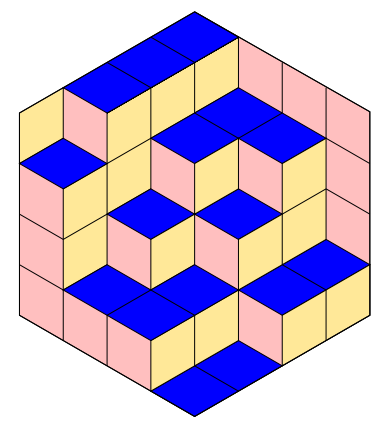

Madeline Brandt \& Amy Wiebe

The slack realization space of a matroid

Volume 2, issue 4 (2019), p. 663-681.

<http://alco.centre-mersenne.org/item/ALCO_2019__2_4_663_0>

(C) The journal and the authors, 2019.

Some rights reserved.

(c) BY This article is licensed under the

Creative Commons ATtribution 4.0 InTERnational License.

http://creativecommons.org/licenses/by/4.0/

Access to articles published by the journal Algebraic Combinatorics on the website http://alco.centre-mersenne.org/ implies agreement with the Terms of Use (http://alco.centre-mersenne.org/legal/).

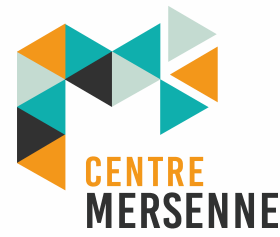

Algebraic Combinatorics is member of the Centre Mersenne for Open Scientific Publishing www.centre-mersenne.org 


\title{
The slack realization space of a matroid
}

\author{
Madeline Brandt \& Amy Wiebe
}

\begin{abstract}
We introduce a new model for the realization space of a matroid, which is obtained from a variety defined by a saturated determinantal ideal, called the slack ideal, coming from the vertex-hyperplane incidence matrix of the matroid. This is inspired by a similar model for the slack realization space of a polytope. We show how to use these ideas to certify nonrealizability of matroids, and describe an explicit relationship to the standard GrassmannPlücker realization space model. We also exhibit a way of detecting projectively unique matroids via their slack ideals by introducing a toric ideal that can be associated to any matroid.
\end{abstract}

\section{INTRODUCTION}

Realization spaces of matroids are well studied objects $[1,2,10]$ which encode not only whether or not the matroid is realizable, but also carry additional information about the structure of the matroid. A realization (or representation) of a rank $d+1$ matroid $M$ is a set of vectors in $\mathbb{k}^{d+1}$ which captures its independence structure. Roughly speaking, a realization space is the set of all such choices of vectors. Fundamental questions in the study of realization spaces of matroids include discovering whether or not a given matroid is realizable, determining over which field it is realizable, finding the structure of the set of realizations, and characterizing when realizations exist. A celebrated theorem of Mnëv states that every semialgebraic set defined over the integers is stably equivalent to the realization space of some oriented matroid. That is, realization spaces of matroids can become arbitrarily complicated. In light of this, we aim to connect the combinatorics of the matroid to properties of its realization space.

Our realization space model will be based off the slack matrix of a matroid. This is a generalization of the slack matrix of a polytope [15], which has been used extensively in the study of extended formulations of polytopes; see for example [7, 13, 15]. In [8] the slack matrix of a polytope was used to construct a realization space for the polytope via its slack ideal. This realization space model and its properties were recently explored in detail in [6]. We extend the results in [6] to matroids both defining the slack realization space of a matroid and examining its properties.

Manuscript received 2nd May 2018, revised 22nd January 2019, accepted 23rd February 2019.

KEYWORDS. matroid, realization space.

ACKnowledgements. M. Brandt was partially supported by the National Science Foundation Graduate Research Fellowship under Grant No. DGE 1106400.

A. Wiebe was partially supported by an NSERC Postgraduate Scholarship. 
In Section 2 we introduce the main objects of study, as well as preliminary results and notation. In Section 3 we discuss two models for the realization space of a matroid. One of our main theorems, Theorem 3.8, shows how the two realization space models can be described via a single overarching variety. In Section 4 we show how the slack realization model can be used to determine whether a matroid has a realization over a certain field. We also reframe the tools of final polynomials [2] in terms of slack ideals, and show how they can be used to improve computational efficiency of realizability checking. In Section 5 we introduce a toric ideal associated to a matroid and study its relationship to the projective uniqueness of the matroid. In Appendix A we include a table of notation used throughout the paper. The computations in this paper are done in Macaulay2 [9] with the help of the Matroids package [3]; code for the computations can be found at http://sites.math. washington. edu/ awiebe.

\section{The SlaCK MATrix of A MATRoid}

Much of this section is analogous to $[6, \S 2]$ to which we refer the reader for further details and excluded proofs. We assume the reader has familiarity with the basic definitions from matroid theory, see [12] or [5]. Throughout the paper, we assume all matroids are simple (having no loops or parallel elements).

Let $\mathbb{k}$ be a field, and $\mathbb{k}^{*}:=\mathbb{k} \backslash\{0\}$. Let $M=(E, \mathcal{B})$ be a matroid of rank $d+1$ with ground set $E=\left\{\mathbf{v}_{1}, \ldots, \mathbf{v}_{n}\right\}$, where each $\mathbf{v}_{i} \in \mathbb{k}^{d+1}$ and $\mathcal{B}$ is its set of bases. If $V$ is the matrix with columns $\mathbf{v}_{1}, \ldots, \mathbf{v}_{n}$, then the independent sets of $M$ are the linearly independent subsets of columns, and we write $M=M[V]$.

Let $\mathcal{H}(M)$ denote the set of hyperplanes of $M$, which are the closed subsets (flats) of rank $d$. In $M[V]$, each hyperplane $H \in \mathcal{H}(M)$ corresponds to a linear subspace of $\mathbb{k}^{d+1}$, so is determined by some linear equation; that is, $H=\left\{\mathbf{x} \in E: \alpha_{H}^{\top} \mathbf{x}=0\right\}$. For $\mathcal{H}(M)=\left\{H_{1}, \ldots, H_{h}\right\}$, let $W$ be the matrix whose columns are the hyperplane defining normals $\alpha_{1}, \ldots, \alpha_{h}$, or some multiple, $\lambda_{j} \alpha_{j}$ for $\lambda_{j} \in \mathbb{k}^{*}$, thereof.

Definition 2.1. A slack matrix of the matroid $M=M[V]$ over $\mathbb{k}$ is an $n \times h$ matrix $S_{M[V]}=V^{\top} W$ for matrices $V$ and $W$ as above.

We wish to parametrize the set of realizations of a matroid by its slack matrices. So, we must determine the characteristics which define the set of all possible slack matrices of a given matroid. We begin by considering the rank of a slack matrix. Given a matrix $S$, its support consists of the entries which are non-zero. Two matrices have the same support if they have the same zero pattern.

Lemma 2.2 (See [6, Lemma 3.1]). If $S$ is a matrix having the same support as a slack matrix of some rank $d+1$ matroid $M=M[V]$, then $\operatorname{rank}(S) \geqslant d+1$.

Corollary 2.3. If $M=M[V]$ is a rank $d+1$ matroid then $\operatorname{rank}\left(S_{M}\right)=d+1$.

Proof. The factored form of $S_{M} \in \mathbb{k}^{n \times(d+1)} \times \mathbb{k}^{(d+1) \times h}$ implies that $\operatorname{rank}\left(S_{M}\right) \leqslant d+1$. The result then follows from Lemma 2.2 .

Now, let $M=(E, \mathcal{B})$ be an abstract matroid of rank $d+1$. Unless otherwise stated, we take $E=[n]=\{1, \ldots, n\}$. A realization of $M$ over $\mathbb{k}$ is a collection of vectors $V=\left\{\mathbf{v}_{1}, \ldots, \mathbf{v}_{n}\right\} \subset \mathbb{k}^{\ell}$ such that the independent subsets of $V$ are indexed by the independent sets of the matroid, so $M=M[V]$. A matroid with a realization is called realizable (also representable, linear or coordinatizable).

Lemma 2.4. The rows of a slack matrix $S_{M}$ form a realization of the matroid $M$.

Proof. It suffices to show that if we label the rows of $S_{M}$ with $[n]$, the subsets indexing linearly independent rows of $S_{M}$ are the independent sets of $M$. Since $S_{M}=V^{T} W$, 
if a subset $J$ of $E$ is dependent, then there exists a vector $\beta \in \mathbb{k}^{n}$ with support indexed by $J$ such that $V \beta=0$. But now, $\beta^{\top} S_{M}=(V \beta)^{\top} W=0$, so $J$ also indexes a dependent subset of the rows of $S_{M}$.

Conversely, suppose $J$ indexes a dependent subset of the rows of $S_{M}$. Then for some $\beta \in \mathbb{k}^{n}$ with support indexed by $J$, we have $0=\beta^{\top} S_{M}=(V \beta)^{\top} W$. Since $W$ has full rank by Corollary 2.3, it must be the case that $V \beta=0$, so that $J$ also indexes a dependent set of $M$.

From now on we assume that realizations come with a fixed labelling of ground set elements and hyperplanes. Then, two slack matrices of the same matroid (which a priori may have had different labellings) now cannot differ by permutations of rows and columns. This also allows us to identify hyperplanes of a realization by their normal vectors or the indices of those vectors. Now, we characterize the set of matrices which correspond to slack matrices of a matroid $M$.

THEOREM 2.5. Let $M$ be a rank $d+1$ matroid with $n$ elements and hyperplanes $\mathcal{H}(M)=\left\{H_{1}, \ldots, H_{h}\right\} . A$ matrix $S \in \mathbb{k}^{n \times h}$ is a slack matrix of some realization of $M$ if and only if both of the following hold:

(i) $\operatorname{supp}(S)=\operatorname{supp}\left(S_{M}\right)$

(ii) $\operatorname{rank}(S)=d+1$.

Proof. Suppose $S$ is a slack matrix of some realization of $M$. Then (i) holds trivially, and (ii) holds by Corollary 2.3.

Conversely, suppose $S$ is a matrix satisfying (i) and (ii). By (ii), $S$ has some rank factorization $S=A B$, where $A \in \mathbb{k}^{n \times(d+1)}$ and $B \in \mathbb{k}^{(d+1) \times h}$. Let $\mathbf{a}_{1}, \ldots, \mathbf{a}_{n} \in \mathbb{k}^{d+1}$ be the rows of $A$ and $\mathbf{b}_{1}, \ldots, \mathbf{b}_{h} \in \mathbb{k}^{d+1}$ be the columns of $B$. Then we claim that the rows of $A$ give a realization of $M$; that is $M=M\left[A^{\top}\right]$. To see this, we show that the hyperplanes of $M$ are also hyperplanes of $M\left[A^{\top}\right]$, and that $M\left[A^{\top}\right]$ can not contain more hyperplanes.

By (i), for each hyperplane $H_{j}$ of $M$, there is a column of $S$ with zeros in positions indexed by elements of $H_{j}$. Since $S=A B$, we have $\mathbf{b}_{j}^{\top} \mathbf{a}_{i}=0$ if and only if $i \in H_{j}$. Thus

$$
\left\{\mathbf{x} \in \mathbb{k}^{d+1}: \mathbf{b}_{j}^{\top} \mathbf{x}=0\right\} \cap\{\operatorname{rows}(A)\}=\left\{\mathbf{a}_{i}\right\}_{i \in H_{j}}
$$

so that $H_{j}$ is also a hyperplane of the matroid $M\left[A^{\top}\right]$.

Now suppose $M\left[A^{\top}\right]$ has an extra hyperplane $H \notin \mathcal{H}(M)$. Let $\left\{i_{1}, \ldots, i_{d}\right\} \subset H$ be any $d$ distinct independent elements of $H$. Since $i_{1}, \ldots, i_{d}$ are also elements of matroid $M$, the flat $\overline{\left\{i_{1}, \ldots, i_{d}\right\}}$ is a hyperplane $H^{\prime}$ of $M$, and thus also a hyperplane of $M\left[A^{\top}\right]$, but $H^{\prime} \neq H$. However, this means that $\left\{i_{1}, \ldots, i_{d}\right\}$ are contained in two distinct hyperplanes of $M\left[A^{\top}\right]$, which is not possible, so we arrive at a contradiction.

Let $\operatorname{GL}(d, \mathbb{k})$ denote the general linear group of degree $d$ over $\mathbb{k}$, that is, the set of $d \times d$ invertible matrices over $\mathbb{k}$. We now recall two equivalence relations on the set of realizations of a matroid $M$, and illustrate how these equivalences are reflected in slack matrices. For $A \in \mathrm{GL}(d+1, \mathbb{k})$, it is easy to check that $V$ and $A V$ define the same matroid. We call these realizations linearly equivalent. If $P \in \mathbb{k}^{n \times n}$ is a permutation matrix which sends $i \mapsto \sigma(i)$, then $V$ and $V P$ define the same matroid up to relabelling the ground set $E=[n]$ with $\sigma(1), \ldots, \sigma(n)$. Thus if $A \in \mathrm{GL}(d+1, \mathbb{k})$ and $B$ is a permutation matrix with any element of $\mathbb{k}^{*}$ in the 1 's positions, then $V, A V B$ define the same matroid. We call the realizations $V, A V B$ projectively equivalent. Call a matroid $M$ projectively unique (over $\mathbb{k}$ ) if all realizations are projectively equivalent. 
LEMMA 2.6. Two realizations of a matroid $M$ are projectively equivalent if and only if their slack matrices are the same up to row and column scaling.

Proof. Suppose we have projectively equivalent representations $U, V$ of $M$. Then $U=$ $A V B$, where $A \in \mathrm{GL}(d+1, \mathbb{k})$ and without loss of generality $B$ is an invertible $n \times n$ diagonal matrix (since we have assumed a fixed labelling of our matroid).

If $H=\left\{\mathbf{v}_{i_{1}}, \ldots, \mathbf{v}_{i_{k}}\right\}$ is a hyperplane of $M[V]$, then $H^{\prime}=\left\{\mathbf{u}_{i_{1}}, \ldots, \mathbf{u}_{i_{k}}\right\}$ is a hyperplane of $M[U]$. Furthermore, if $\alpha_{H} \in \mathbb{k}^{d+1}$ is normal to $H$, then since $\mathbf{u}_{i}=A \mathbf{v}_{i} \cdot b_{i}$, $A^{-\top} \alpha_{H}$ is normal to $H^{\prime}$, so that a slack matrix of $M[U]$ is

$$
S_{M[U]}=U^{\top}\left[A^{-\top} \alpha_{H}\right]_{H \in \mathcal{H}}=B^{\top} V^{\top} A^{\top}\left[A^{-\top} \alpha_{H}\right]_{H \in \mathcal{H}}=B^{\top} V^{\top} W=B^{\top} S_{M[V]} .
$$

Since we can always scale columns of a slack matrix, this completes the proof.

Conversely, suppose we have realizations $U$ and $V$ of the matroid $M$ such that $S_{M[U]}=D_{n} S_{M[V]} D_{h}$ for invertible diagonal matrices $D_{n} \in \mathbb{k}^{n \times n}, D_{h} \in \mathbb{k}^{h \times h}$. By definition, $S_{M[U]}=U^{\top} W$ and $S_{M[V]}=V^{\top} W^{\prime}$. Multiplying both sides of the above equation on the right by the right inverse $Y$ of matrix $W$, we find

$$
U^{\top}=D_{n} V^{\top} W^{\prime} D_{h} Y
$$

We see that $W^{\prime} D_{h} Y$ is a $(d+1) \times(d+1)$ invertible matrix, which makes $U$ and $V$ projectively equivalent, as desired.

LEMMA 2.7. Two realizations of a matroid $M$ are linearly equivalent if and only if their slack matrices are the same up to column scaling.

Proof. By taking $B, D_{n}$ each to be the $n \times n$ identity matrix in the proof of Lemma 2.6, we obtain the desired result.

We now define an analog of the slack matrix which can be constructed for any abstract matroid, even ones which are not realizable, as follows.

DEFINITION 2.8. Define the symbolic slack matrix of matroid $M$ to be the matrix $S_{M}(\mathbf{x})$ with rows indexed by elements $i \in E$, columns indexed by hyperplanes $H_{j} \in$ $\mathcal{H}(M)$ and $(i, j)$-entry

$$
S_{M}(\mathbf{x})_{i j}= \begin{cases}x_{i j} & \text { if } i \notin H_{j} \\ 0 & \text { if } i \in H_{j} .\end{cases}
$$

The slack ideal of $M$ is the saturation of the ideal generated by the $(d+2)$-minors of $S_{M}(\mathbf{x})$, namely

$$
I_{M}:=\left\langle(d+2)-\text { minors of } S_{M}(\mathbf{x})\right\rangle:\left(\prod_{i=1}^{n} \prod_{j: i \notin H_{j}} x_{i j}\right)^{\infty} \subset \mathbb{k}[\mathbf{x}] .
$$

Suppose there are $t$ variables in $S_{M}(\mathbf{x})$. The slack variety is the variety $\mathcal{V}\left(I_{M}\right) \subset \mathbb{k}^{t}$. The saturation of $I_{M}$ by the product of all the variables guarantees that there are no components of $\mathcal{V}\left(I_{M}\right)$ that live entirely in coordinate hyperplanes. If $\mathbf{s} \in \mathbb{k}^{t}$ is a zero of $I_{M}$, then we identify it with the matrix $S_{M}(\mathbf{s})$.

EXAmple 2.9. Consider the rank 3 matroid $M_{4}=M[V]$ for $V$ whose columns are $\mathbf{v}_{1}=(-2,-2,1)^{\top}, \mathbf{v}_{2}=(-1,1,1)^{\top}, \mathbf{v}_{3}=(0,4,1)^{\top}, \mathbf{v}_{4}=(2,-2,1)^{\top}, \mathbf{v}_{5}=(1,1,1)^{\top}$, $\mathbf{v}_{6}=(0,0,1)^{\top}$. Projecting onto the plane $z=1$, this can be visualized as the points of intersection of four lines in the plane, as in Figure 1. 


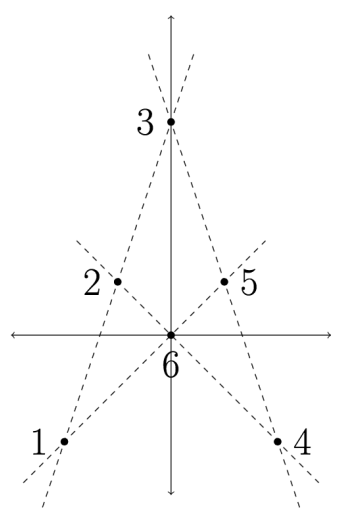

\begin{tabular}{|c|c|c|c|c|c|c|c|}
\hline & $H_{1}$ & $H_{2}$ & $H_{3}$ & $H_{4}$ & $H_{5}$ & $H_{6}$ & $H_{7}$ \\
\hline & 123 & 246 & 345 & 156 & 25 & 14 & 36 \\
\hline 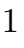 & -0 & $x_{12}$ & $x_{13}$ & 0 & $x_{15}$ & 0 & $x_{17}$ \\
\hline & 0 & 0 & $x_{23}$ & $x_{24}$ & 0 & $x_{26}$ & $x_{27}$ \\
\hline & 0 & $x_{32}$ & 0 & $x_{34}$ & $x_{35}$ & $x_{36}$ & 0 \\
\hline & $x_{41}$ & 0 & 0 & $x_{44}$ & $x_{45}$ & 0 & $x_{47}$ \\
\hline & $x_{51}$ & $x_{52}$ & 0 & 0 & 0 & $x_{56}$ & $x_{57}$ \\
\hline & $x_{61}$ & 0 & $x_{63}$ & 0 & $x_{65}$ & $x_{66}$ & \\
\hline
\end{tabular}

Figure 1. The point-line configuration $M_{4}$ of Example 2.9, and its symbolic slack matrix $S_{M_{4}}(\mathbf{x})$.

A slack matrix for this realization is then

$$
\begin{aligned}
& S_{M_{4}}=\left[\begin{array}{ccc}
-2 & -2 & 1 \\
-1 & 1 & 1 \\
0 & 4 & 1 \\
2 & -2 & 1 \\
1 & 1 & 1 \\
0 & 0 & 1
\end{array}\right]\left[\begin{array}{ccccccc}
H_{1} & H_{2} & H_{3} & H_{4} & H_{5} & H_{6} & H_{7} \\
123 & 246 & 345 & 156 & 25 & 14 & 36 \\
1 & 3 & 6 & -3 & 0 & 0 & 4 \\
1 & 3 & 2 & 3 & 2 & 4 & 0 \\
-4 & 0 & -8 & 0 & -2 & 8 & 0
\end{array}\right] \\
& \begin{array}{lllllll}
H_{1} & H_{2} & H_{3} & H_{4} & H_{5} & H_{6} & H_{7}
\end{array} \\
& \begin{array}{lllllll}
123 & 246 & 345 & 156 & 25 & 14 & 36
\end{array} \\
& \begin{array}{rl}
1 & 1 \\
2 & 3 \\
& 4 \\
5 & 6
\end{array}\left[\begin{array}{ccccccc}
0 & -12 & -24 & 0 & -6 & 0 & -8 \\
0 & 0 & -12 & 6 & 0 & 12 & -4 \\
0 & 12 & 0 & 12 & 6 & 24 & 0 \\
-12 & 0 & 0 & -12 & -6 & 0 & 8 \\
-6 & 6 & 0 & 0 & 0 & 12 & 4 \\
-4 & 0 & -8 & 0 & -2 & 8 & 0
\end{array}\right],
\end{aligned}
$$

where using $\left\{\mathbf{v}_{j_{1}}, \ldots, \mathbf{v}_{j_{d}}\right\} \subset H$ independent, we calculate each $\alpha_{H}$ as

$$
\operatorname{det}\left[\begin{array}{cccc}
\widehat{e_{1}} & \mid & \cdots & \mid \\
\vdots & \mathbf{v}_{j_{1}} & \cdots & \mathbf{v}_{j_{d}} \\
\widehat{e_{d+1}} & \mid & \cdots & \mid
\end{array}\right] .
$$

The symbolic slack matrix of $M_{4}$ is in Figure 1. We take the ideal of 4-minors of this matrix, and saturate with respect to the product of all of the variables to get the slack ideal $I_{M_{4}}$. This has codimension 12, degree 293 and is generated by the 72 binomial generators in Table 1. In Section 5 we will see these correspond to the 72 cycles in the bipartite non-incidence graph of this configuration (Figure 6).

REMARK 2.10. In [6], given a set of $n$ vertices $V \subset \mathbb{k}^{d}$ defining a $d$-polytope $P=$ $\operatorname{conv}(V)$, they include only the facet defining hyperplanes in the slack matrix. We can also form a matroid associated to this polytope by considering all the hyperplanes; that is, we define the matroid $M=M\left[V^{\prime}\right]$ where $V^{\prime} \subset \mathbb{k}^{(d+1) \times n}$ is the matrix obtained from $V$ by appending a 1 to each vector. Then the symbolic slack matrix of $P$ defined in [6] is the restriction of the symbolic slack matrix of matroid $M$ to the subset 


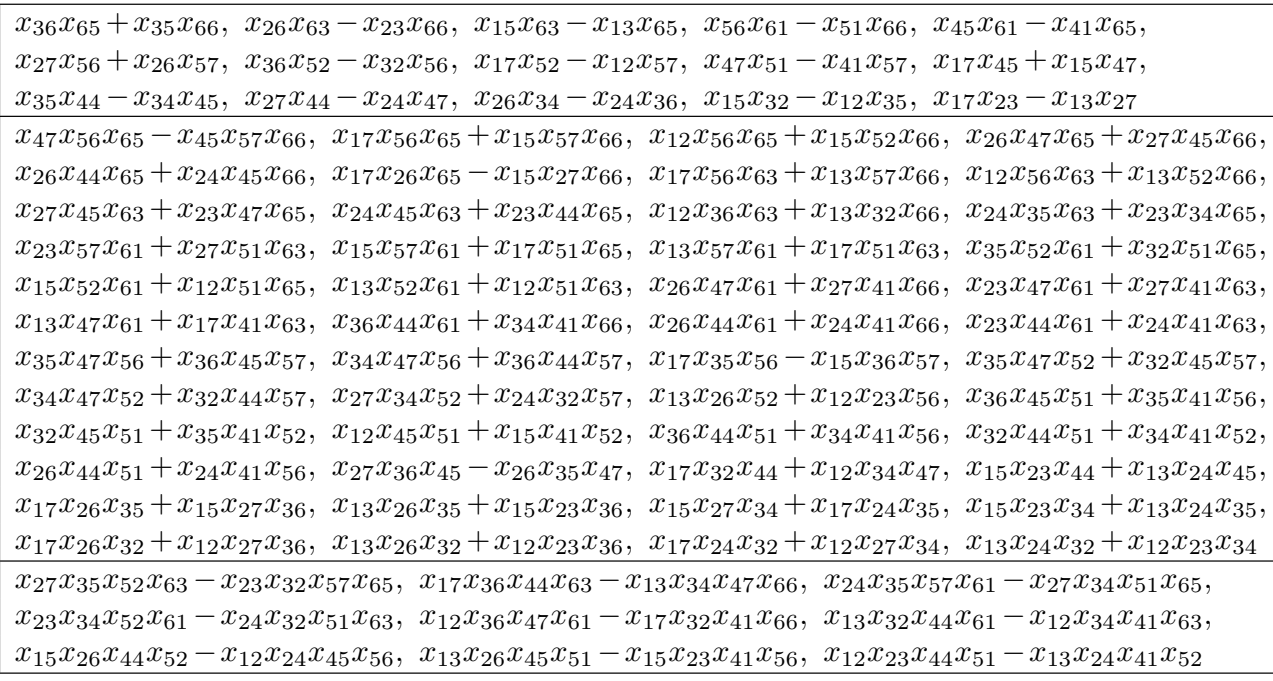

TABLE 1. The 72 generators of $I_{M_{4}}$.

of columns corresponding to facet-defining hyperplanes. Thus the slack ideal of the polytope is always contained in the slack ideal of the matroid, $I_{P} \subseteq I_{M}$. We illustrate with the following example.

EXAMPLE 2.11. We consider the triangular prism $P$ labelled as in Figure 2. As a 3 -polytope, its facets are given by the hyperplanes 1234, 1256, 3456, 135, 246 and the symbolic slack matrix is in Figure 2. Its slack ideal $I_{P}$ is generated by three binomials.

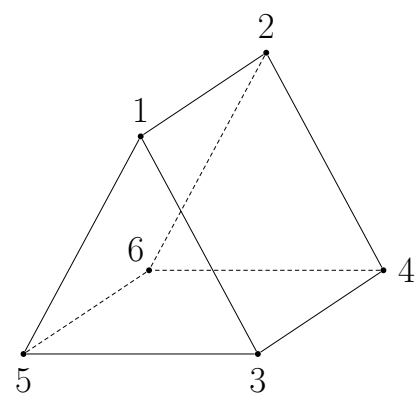

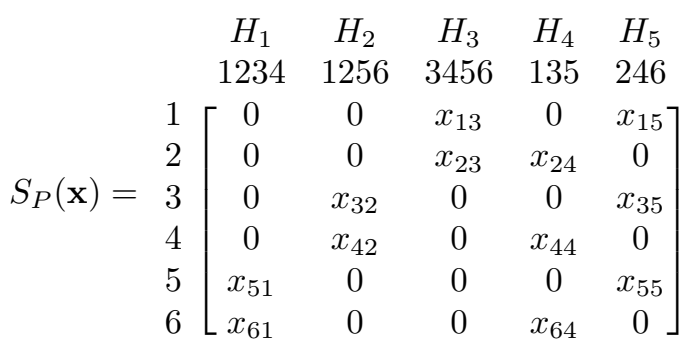

Figure 2. The triangular prism $P$ of Example 2.11 and its symbolic slack matrix as a polytope.

Considering $P$ as a rank 4 matroid which has the three facets $1234,1256,3456$ of $P$ as its non-bases, we obtain following symbolic slack matrix.

$$
\begin{aligned}
& \begin{array}{lllll|llllll}
H_{1} & H_{2} & H_{3} & H_{4} & H_{5} & H_{6} & H_{7} & H_{8} & H_{9} & H_{10} & H_{11}
\end{array} \\
& \begin{array}{lllll|llllll}
1234 & 1256 & 3456 & 135 & 246 & 136 & 146 & 145 & 245 & 235 & 236
\end{array} \\
& S_{M}(\mathbf{x})=\begin{array}{l}
1 \\
2 \\
3 \\
4 \\
5 \\
6
\end{array}\left[\begin{array}{ccccc|cccccc}
0 & 0 & x_{13} & 0 & x_{15} & 0 & 0 & 0 & x_{19} & x_{1,10} & x_{1,11} \\
0 & 0 & x_{23} & x_{24} & 0 & x_{26} & x_{27} & x_{28} & 0 & 0 & 0 \\
0 & x_{32} & 0 & 0 & x_{35} & 0 & x_{37} & x_{38} & x_{39} & 0 & 0 \\
0 & x_{42} & 0 & x_{44} & 0 & x_{46} & 0 & 0 & 0 & x_{4,10} & x_{4,11} \\
x_{51} & 0 & 0 & 0 & x_{55} & x_{56} & x_{57} & 0 & 0 & 0 & x_{5,11} \\
x_{61} & 0 & 0 & x_{64} & 0 & 0 & 0 & x_{68} & x_{69} & x_{6,10} & 0
\end{array}\right]
\end{aligned}
$$


Not only is $I_{P} \subseteq I_{M}$ but in this case $I_{P}$ is the elimination ideal given by eliminating the variables in the columns indexed by the additional hyperplanes $H_{6}, \ldots, H_{11}$.

\section{Realization SPACE MOdEls}

A realization space for a rank $d+1$ matroid $M$ with $n$ elements is, roughly speaking, a space whose points are in correspondence with (equivalence classes of) collections of vectors $V=\left\{\mathbf{v}_{1}, \ldots, \mathbf{v}_{n}\right\} \subset \mathbb{k}^{d+1}$ whose matroid $M[V]$ is $M$. In this section we show that the slack variety defined in Section 2 provides such a realization space, and we relate it to another realization space called the Grassmannian of the matroid.

Theorem 2.5 characterizes the slack matrices of realizations of a matroid. The next theorem shows that the slack variety captures exactly these matrices.

THEOREM 3.1. Let $M$ be a rank $d+1$ matroid. Then $V$ is a realization of $M$ if and only if $S_{M[V]}=S_{M}(\mathbf{s})$ where $\mathbf{s} \in \mathcal{V}\left(I_{M}\right) \cap\left(\mathbb{k}^{*}\right)^{t}$.

Proof. Let $V$ be a realization of $M$. Then $S_{M[V]}=S_{M}(\mathbf{s})$ for some $\mathbf{s} \in\left(\mathbb{k}^{*}\right)^{t}$ by Theorem 2.5 (i). Furthermore, $\operatorname{rank}\left(S_{M[V]}\right)=d+1$ by Corollary 2.3 , so that its $(d+2)$-minors vanish and thus $\mathbf{s} \in \mathcal{V}\left(I_{M}\right)$, as desired.

Let $V \in \mathbb{k}^{(d+1) \times n}$ be such that $S_{M[V]}=S_{M}(\mathbf{s})$ for some $\mathbf{s} \in \mathcal{V}\left(I_{M}\right) \cap\left(\mathbb{k}^{*}\right)^{t}$. Then $\operatorname{supp}\left(S_{M[V]}\right)=\operatorname{supp}\left(S_{M}\right)$ and $\operatorname{rank}\left(S_{M[V]}\right) \leqslant d+1$. But now by Lemma 2.2, $\operatorname{rank}\left(S_{M[V]}\right) \geqslant d+1$, making $V$ a realization of $M$ by Theorem 2.5 .

Since we know that the set of realizations of a matroid is closed under row and column scalings, Theorem 3.1 implies the following corollary. We denote the torus of row and column scalings, $\left(\mathbb{k}^{*}\right)^{n} \times\left(\mathbb{k}^{*}\right)^{h}$, by $T_{n, h}$.

COROLLARY 3.2. The slack variety is closed under the action of the group $T_{n, h}$, where $\left(\mathbb{k}^{*}\right)^{n}$ acts by row scaling (left multiplication by diagonal matrices) and $\left(\mathbb{k}^{*}\right)^{h}$ acts by column scaling (right multiplication by diagonal matrices).

Theorem 3.1 and Corollary 3.2 tell us that the slack variety is a realization space for matroid $M$ and the slack variety modulo the action of $T_{n, h}$ is a realization space for the projective equivalence classes of realizations of $M$.

Definition 3.3. The slack realization space of a rank $d+1$ matroid $M$ on $n$ elements with $h$ hyperplanes is the image of the slack variety inside a product of projective spaces $\mathcal{V}\left(I_{M}\right) \cap\left(\mathbb{k}^{*}\right)^{t} \hookrightarrow\left(\mathbb{P}^{n-1}\right)^{h}$, where $\mathbf{s}$ is sent to the columns of $S_{M}(\mathbf{s})$.

Proposition 3.4. Let $M$ be a rank $d+1$ matroid on $n$ elements with h hyperplanes. Then the points of its slack realization space are in one-to-one correspondence with linear equivalence classes of realizations of $M$.

Proof. Under this embedding, two slack matrices which differ by column scaling are the same point in $\left(\mathbb{P}^{n-1}\right)^{h}$. So, the result follows by Lemma 2.7 .

Next we describe a known model for the realization space of a matroid arising from a subvariety of a Grassmannian. The Grassmannian $\operatorname{Gr}(d+1, n)$ is a variety whose points correspond to $(d+1)$-dimensional linear subspaces of a fixed $n$-dimensional vector space $\Lambda$. It embeds into $\mathbb{P}_{\left(\begin{array}{c}n \\ d+1\end{array}\right)-1}$ as follows. Any $(d+1)$-dimensional linear subspace of $\Lambda$ can be described as the row space of a $(d+1) \times n$ matrix of rank $d+1$. However, two matrices $A$ and $B$ have the same row space when there is a matrix $G \in \mathrm{GL}(d+1, \mathbb{k})$ such that $A=G B$. Thus, to ensure that we have a one-toone correspondence between subspaces and points in the Grassmannian, we record a $(d+1) \times n$ matrix by its vector of $(d+1)$-minors. We call this the Plücker vector, and it has coordinates indexed by subsets $\sigma$ of $[n]$ of size $d+1$. The Plücker ideal 
$P_{d+1, n} \subseteq \mathbb{k}[\mathbf{p}]=\mathbb{k}\left[p_{\sigma}|\sigma \subset[n],| \sigma \mid=d+1\right]$ is the set of all polynomials which vanish on every vector of $(d+1)$-minors coming from some $(d+1) \times n$ matrix. It is generated by the homogeneous quadratic Plücker relations and cuts out the Grassmannian as a variety inside $\mathbb{P}\left(\begin{array}{c}n \\ d+1\end{array}\right)-1$.

If we have a rank $d+1$ matroid $M=(E, \mathcal{B})$ with realization $V \in \mathbb{k}^{(d+1) \times n}$, the Plücker coordinate $p_{\sigma}$ of $V$ is zero if and only if $\sigma$ is a dependent set of $M$. Thus, realizations of $M$ correspond to the subvariety of $\operatorname{Gr}(d+1, n)$ defined by setting the Plücker coordinates of non-bases to 0 . This variety is also cut out by an ideal, namely, the Grassmannian ideal $P_{M} \subset \mathbb{k}\left[\mathbf{p}_{\mathcal{B}}\right]$ of $M$,

$$
P_{M}:=\left(P_{d+1, n}+\left\langle p_{\sigma}: \sigma \notin \mathcal{B}\right\rangle\right) \cap \mathbb{k}\left[\mathbf{p}_{\mathcal{B}}\right],
$$

where $\mathbb{k}\left[\mathbf{p}_{\mathcal{B}}\right]:=\mathbb{k}\left[p_{\sigma} \mid \sigma\right.$ is a basis of $\left.M\right]$ is the polynomial ring with one variable for each basis $\sigma \in \mathcal{B}$. This is the ideal obtained from $P_{d+1, n} \subset \mathbb{k}[\mathbf{p}]$ by setting the variables indexed by non-bases of $M$ to 0 .

Definition 3.5. The Grassmannian of $M$, denoted $\operatorname{Gr}(M)$, is $\mathcal{V}\left(P_{M}\right) \cap\left(\mathbb{k}^{*}\right)^{|\mathcal{B}|}$. The points in $\operatorname{Gr}(M)$ correspond to $\mathrm{GL}(d+1, \mathbb{k})$ equivalence classes of $(d+1) \times n$ matrices which realize the matroid $M$.

3.1. Universal Realization ideal. Given a matroid $M$, we now define an ideal whose variety contains pairs $(\mathbf{s}, \mathbf{q})$, where $\mathbf{q}$ is a Plücker vector and $\mathbf{s}$ the nonzero entries of a slack matrix, and both come from the same realization of $M$.

If $V$ is a realization of a rank $d+1$ matroid $M=(E, \mathcal{B})$, then a slack matrix $S_{M[V]}$ can be filled with the Plücker coordinates of $V$, which can be seen from (1). Given a hyperplane $H_{j} \in \mathcal{H}(M)$, we record all possible substitutions of Plücker variables for slack variables using a matrix $M_{H_{j}}$ whose rows are indexed by $i \in E \backslash H_{j}$, and whose columns are indexed by subsets $J=\left\{j_{1}, \ldots, j_{d}\right\} \subset E$ with $\bar{J}=H$; that is,

$$
M_{H_{j}}=\left[\begin{array}{cccc}
x_{i_{1} j} & \operatorname{sgn}\left(i_{1}, J_{1}\right) \cdot p_{i_{1} \cup J_{1}} & \cdots & \operatorname{sgn}\left(i_{1}, J_{k}\right) \cdot p_{i_{1} \cup J_{k}} \\
\vdots & \vdots & \cdots & \vdots \\
x_{i_{m} j} \operatorname{sgn}\left(i_{m}, J_{1}\right) \cdot p_{i_{m} \cup J_{1}} & \cdots & \operatorname{sgn}\left(i_{m}, J_{k}\right) \cdot p_{i_{m} \cup J_{k}}
\end{array}\right] \text {, }
$$

where $\operatorname{sgn}(i, J)$ is the sign of the permutation putting $\left(i, j_{1}, \ldots, j_{d}\right)$ in increasing order.

EXAMPLE 3.6. Recall the matroid $M_{4}$ of Example 2.9 pictured in Figure 1. Consider the hyperplane $H_{2}=246$. It corresponds to slack variables $x_{i 2}$ for $i=1,3,5$ and its independent subsets are 24,26 , and 46 . So the matrix $M_{246}$ has the form

$$
M_{246}=\left[\begin{array}{rrrr}
x_{12} & p_{124} & p_{126} & p_{146} \\
x_{32} & -p_{234} & -p_{236} & p_{346} \\
x_{52} & p_{245} & -p_{256} & -p_{456}
\end{array}\right] \text {. }
$$

Definition 3.7. Let $M=(E, \mathcal{B})$ be a matroid, $P_{M}$ be the Grassmannian ideal of $M$ and $I_{2}\left(M_{H_{j}}\right)$ be the ideal of 2-minors of the matrix $M_{H_{j}}$. The universal realization ideal of $M$ is

$$
U_{M}:=P_{M}+\sum_{H_{j} \in \mathcal{H}(M)} I_{2}\left(M_{H_{j}}\right) \subseteq \mathbb{k}[\mathbf{x}, \mathbf{p}] .
$$

Intuitively, insisting that the matrices $M_{H_{j}}$ have rank 1 corresponds to ensuring the columns of the slack matrix are simply scaled versions of the appropriate Plücker coordinates. We now state the main result of this section.

TheOREM 3.8. Let $M=([n], \mathcal{B})$ be a rank $d+1$ matroid with universal realization ideal $U_{M} \subseteq \mathbb{k}[\mathbf{x}, \mathbf{p}]$. Then $\mathcal{V}\left(U_{M}\right) \in \mathbb{k}^{t} \times \mathbb{k}^{|\mathcal{B}|}$ satisfies 
(i) the projection of $\mathcal{V}\left(U_{M}\right)$ onto the Plücker coordinates, $\pi_{\mathbf{p}}: \mathcal{V}\left(U_{M}\right) \rightarrow \mathbb{k}^{|\mathcal{B}|}$, is the Grassmannian of the matroid,

$$
\overline{\pi_{\mathbf{p}}\left(\mathcal{V}\left(U_{M}\right)\right)}=\overline{\operatorname{Gr}(M)} ;
$$

(ii) the projection of $\mathcal{V}\left(U_{M}\right) \cap\left(\left(\mathbb{k}^{*}\right)^{t} \times\left(\mathbb{k}^{*}\right)^{|\mathcal{B}|}\right)$ onto the slack coordinates, $\pi_{\mathbf{x}}$ : $\mathcal{V}\left(U_{M}\right) \rightarrow \mathbb{k}^{t}$, is the set of slack matrices of realizations of $M$,

$$
\pi_{\mathbf{x}}\left(\mathcal{V}\left(U_{M}\right) \cap\left(\left(\mathbb{k}^{*}\right)^{t} \times\left(\mathbb{k}^{*}\right)^{|\mathcal{B}|}\right)\right)=\mathcal{V}\left(I_{M}\right) \cap\left(\mathbb{k}^{*}\right)^{t}
$$

The proof of this theorem requires several preliminary lemmas. We first have the following result on Gröbner bases. (For notation and further details see [4].)

LEMmA 3.9. Fix an elimination order on $\mathbb{k}[\mathbf{x}, \mathbf{p}]$. Given two $\mathbf{x}$-homogeneous polynomials $f, g$ and an $\mathbf{x}$-homogeneous set $\mathcal{G} \subset \mathbb{k}[\mathbf{x}, \mathbf{p}]$, if $h:=\overline{S(f, g)}$ Gith $h \neq 0$, then $\operatorname{deg}_{\mathbf{x}}(h) \geqslant \max \left\{\operatorname{deg}_{\mathbf{x}}(f), \operatorname{deg}_{\mathbf{x}}(g)\right\}$.

LEmma 3.10. The Grassmannian ideal of a matroid can be obtained by eliminating the slack variables from its universal realization ideal. That is, $P_{M}=U_{M} \cap \mathbb{k}[\mathbf{p}]$.

Proof. We obtain one containment by the definition of $U_{M}$, since

$$
P_{M}=P_{M} \cap \mathbb{k}[\mathbf{p}] \subseteq\left(P_{M}+\sum_{H \in \mathcal{H}(M)} I_{2}\left(M_{H}\right)\right) \cap \mathbb{k}[\mathbf{p}]=U_{M} \cap \mathbb{k}[\mathbf{p}] .
$$

It remains to show the reverse containment. Fix an elimination order on $\mathbb{k}[\mathbf{x}, \mathbf{p}]$ eliminating the $\mathbf{x}$ variables. Let $\mathcal{G}$ be a Gröbner basis for $U_{M}$ with respect to this ordering. Then, it suffices to show that

$$
\mathcal{G} \cap \mathbb{k}[\mathbf{p}] \subset P_{M}
$$

If we start with a generating set satisfying (2), then by Lemma 3.9, any terms which are added to $\mathcal{G}$ after applying each step of Buchberger's algorithm with $\mathbf{x}$-degree 0 must be the reduction of an $S$-pair of elements which also have x-degree 0 , and are therefore also contained in $P_{M}$.

It remains to show that an initial generating set of $U_{M}$ satisfies (2). Taking the generating set of the definition, it is enough to show that any minor in $I_{2}\left(M_{H}\right)$ not containing a slack variable is already in $P_{M}$. It is not hard to check that any such minor already arises in $P_{M}$ as some 3 -term Plücker relation having a term $p_{\sigma} p_{\tau}$ for some $\sigma \notin \mathcal{B}$ which gets set to zero.

Proof of Theorem 3.8.

(i) This follows from the definition of $\operatorname{Gr}(M)$ and Lemma 3.10.

(ii, $\subset)$ Let $(\mathbf{s}, \mathbf{q}) \in \mathcal{V}\left(U_{M}\right) \cap\left(\left(\mathbb{k}^{*}\right)^{t} \times\left(\mathbb{k}^{*}\right)^{|\mathcal{B}|}\right)$. From q we obtain a $(d+1) \times n$ matrix $V$ with Plücker vector whose nonzero coordinates come from $\mathbf{q}$. We claim that $V$ is a realization of $M$, so that $S_{M[V]} \in \mathcal{V}\left(I_{M}\right) \cap\left(\mathbb{k}^{*}\right)^{t}$ by Theorem 3.1. Furthermore, we claim that $S_{M[V]}$ and $S_{M}(\mathbf{s})$ are the same up to column scaling, so that $\mathbf{s} \in \mathcal{V}\left(I_{M}\right) \cap\left(\mathbb{k}^{*}\right)^{t}$ by Corollary 3.2 .

A subset of $d+1$ columns of $V$ is independent if and only if the corresponding Plücker coordinate is nonzero. Since $\mathbf{q} \in\left(\mathbb{k}^{*}\right)^{|\mathcal{B}|}$, the nonzero Plücker coordinates correspond to bases $B \in \mathcal{B}$ of $M$. Hence $V$ is a realization of $M$.

Fix a hyperplane $H$ and an independent subset $J=\left\{j_{1}, \ldots, j_{d}\right\} \subset H$. Then the first column of $M_{H}$ is $\left(s_{i H}\right)_{i \notin H}^{\top}$, column $J$ is $\left(\operatorname{sgn}(i, J) \cdot q_{i \cup J}\right)_{i \notin H}^{\top}$, and by definition of $U_{M}$, there exists $\lambda_{H} \in \mathbb{k}^{*}$ such that $s_{i H}=\lambda_{H} \operatorname{sgn}(i, J) \cdot q_{i \cup J}$ for all $i$. Since $\mathbf{q}$ is the Plücker vector of $V$, this gives

$$
s_{i H}=\lambda_{H} \operatorname{sgn}(i, J) \cdot q_{i \cup J}=\lambda_{H} \operatorname{det}\left(\mathbf{v}_{i}, \mathbf{v}_{j_{1}}, \ldots, \mathbf{v}_{j_{d}}\right), \quad \forall i
$$


so that using subset $J$ to calculate $\alpha_{H}$, and hence the entries of $S_{M[V]}$, as in Example 2.9, we see that $S_{M[V]} D_{h}=S_{M}(\mathbf{s})$, for $D_{h}$ the diagonal matrix with entries $\lambda_{H}$.

(ii, つ) Let $\mathbf{s} \in \mathcal{V}\left(I_{M}\right) \cap\left(\mathbb{k}^{*}\right)^{t}$. By Theorem 3.1, $S_{M}(\mathbf{s})$ is a slack matrix of some realization $V$ of $M$. Let $\mathbf{q}$ be the vector of Plücker coordinates of $V$. We claim $(\mathbf{s}, \mathbf{q}) \in \mathcal{V}\left(U_{M}\right) \cap\left(\left(\mathbb{k}^{*}\right)^{t} \times\left(\mathbb{k}^{*}\right)^{|\mathcal{B}|}\right)$. To see this it suffices to show that the columns of $M_{H}$, with entries defined using $(\mathbf{s}, \mathbf{q})$, are scalar multiples of each other for each $H \in \mathcal{H}(M)$; that is, the $I_{2}\left(M_{H}\right)$ ideals are satisfied, since the Plücker coordinates necessarily satisfy the Plücker ideal equations, and hence the equations of $P_{M}$.

The $(i, H)$ slack entry $s_{i H}$ is of the form $\operatorname{det}\left(\mathbf{v}_{i}, \mathbf{w}_{1}, \ldots, \mathbf{w}_{d}\right)$ for some choice of $\mathbf{w}_{1}, \ldots, \mathbf{w}_{d}$ which span the hyperplane $H$. Each subsequent column of $M_{H}$ has entries $\operatorname{det}\left(\mathbf{v}_{i}, \mathbf{v}_{j_{1}}, \ldots, \mathbf{v}_{j_{d}}\right)$, obtained from $\mathbf{q}$ as above, for $j_{1}<\cdots<j_{d}$ spanning $H$. Since each $\mathbf{v}_{j_{k}}$ lies on the hyperplane $H$, there is a sequence of elementary column operations that takes the matrix with columns $\mathbf{v}_{i}, \mathbf{w}_{1}, \ldots, \mathbf{w}_{d}$ to the one with columns $\mathbf{v}_{i}, \mathbf{v}_{j_{1}}, \ldots, \mathbf{v}_{j_{d}}$ for each $i$. These column operations change the determinant by some scale factor $\lambda \in \mathbb{k}^{*}$ for all $i$, so that each column of $M_{H}$ is a scalar multiple of the first column of slack entries as required.

Corollary 3.11. Let $M=(E, \mathcal{B})$ be a rank $d+1$ matroid. Then

$$
\sqrt{I_{M}}=\sqrt{U_{M}:\left(\prod_{i \in E, H \in \mathcal{H}} x_{i H} \prod_{\sigma \in \mathcal{B}} p_{\sigma}\right)^{\infty} \cap \mathbb{k}[\mathbf{x}] .}
$$

By universality [10], we do not expect that $I_{M}$ is radical for every matroid. Thus, Corollary 3.11 may be the strongest relationship between $I_{M}$ and $U_{M}$.

EXAMPLE 3.12. We now continue Example 2.9, for which we can verify Theorem 3.8 at the level of ideals. Let $P_{M_{4}}$ be the Grassmannian ideal of this matroid, which is generated by the Plücker relations with variables $p_{123}, p_{246}, p_{345}, p_{156}$ set to 0 . Let $I$ be the ideal which guarantees each of the seven matrices $M_{123}, M_{246}, M_{345} M_{156}$, $M_{25}, M_{14}, M_{36}$ have rank 1 ; that is, $I=\sum_{H \in \mathcal{H}\left(M_{4}\right)} I_{2}\left(M_{H}\right)$. Then, the universal realization ideal is $U_{M}=I+P_{M_{4}}$. In this case, we can compute that $P_{M}=U_{M} \cap \mathbb{k}[\mathbf{p}]$ and $I_{M}=U_{M}:\left(\prod x_{i H} \prod p_{\sigma}\right)^{\infty} \cap \mathbb{k}[\mathbf{x}]$.

\section{NON-REALIZABILITY}

In this section we illustrate how the slack ideal can be used to determine matroid realizability over a given field. This is a well-studied problem $[1,2,10]$ for which a complete characterization is only known in a very limited number of cases. Observe that Theorem 3.1 gives us the following criterion for realizability.

Corollary 4.1. A matroid $M$ is realizable over $\mathbb{k}$ if and only if $\mathcal{V}\left(I_{M}\right) \cap\left(\mathbb{k}^{*}\right)^{t} \neq \varnothing$.

We now recast this into a test for realizability in terms of the slack ideal.

Proposition 4.2. Let $M$ be an abstract matroid and $\mathbb{k}$ be a field. If the slack ideal $I_{M}=\langle 1\rangle$ over $\mathbb{k}$, then $M$ is not realizable over $\mathbb{k}$. If $\mathbb{k}$ is algebraically closed and $M$ is not realizable over $\mathbb{k}$, then $I_{M}=\langle 1\rangle$.

Proof. If $M$ is realizable over $\mathbb{k}$, then there exists a slack matrix $S_{M}$ which is an element of the variety $\mathcal{V}\left(I_{M}\right)$ by Theorem 3.1. Then we cannot have $I_{M}=\langle 1\rangle$. On 
the other hand, if $I_{M} \neq\langle 1\rangle$, then $\mathcal{V}\left(I_{M}\right)$ is not empty by the Nullstellensatz, and since $I_{M}$ is saturated with respect to the product of the variables, $\mathcal{V}\left(I_{M}\right)$ is not contained entirely in the coordinate hyperplanes. Therefore, by Theorem 3.1 there is a slack matrix $S_{M}$, and the rows of $S_{M}$ give a realization of $M$ by Lemma 2.4.

ExAmple 4.3. Consider the Fano plane $M_{F}$. It is a rank 3 matroid on 7 elements $E=\{0,1,2,3,4,5,6\}$, depicted in Figure 3 with its symbolic slack matrix. It is known that $M_{F}$ is only realizable in characteristic 2. Using Macaulay2 [9], we find $I_{M_{F}}=\langle 1\rangle$ over $\mathbb{Q}$. So, the Fano plane is not realizable over $\mathbb{Q}$ by Proposition 4.2 .

Over $\mathbb{F}_{2}$, the slack ideal is generated by 126 binomials of degrees 2,3 , and 4 . So, setting all variables to 1 in the slack matrix gives the realization of $M_{F}$ in $\mathbb{F}_{2}^{7}$.

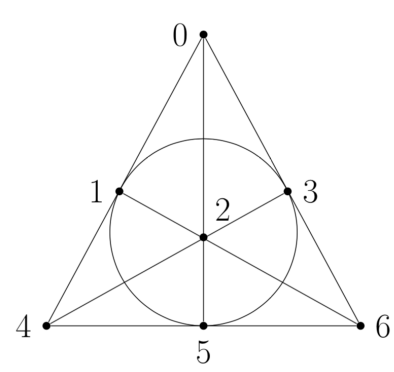

\begin{tabular}{|c|c|c|c|c|c|c|c|}
\hline & $H_{1}$ & $H_{2}$ & $H_{3}$ & $H_{4}$ & $H_{5}$ & $H_{6}$ & $H_{7}$ \\
\hline & 126 & 014 & 456 & 025 & 036 & 234 & 135 \\
\hline 0 & {$\left[x_{01}\right.$} & 0 & $x_{03}$ & 0 & 0 & $x_{06}$ & $\left.x_{07}\right]$ \\
\hline 1 & 0 & 0 & $x_{13}$ & $x_{14}$ & $x_{15}$ & $x_{16}$ & 0 \\
\hline 2 & 0 & $x_{22}$ & $x_{23}$ & 0 & $x_{25}$ & 0 & $x_{27}$ \\
\hline 3 & $x_{31}$ & $x_{32}$ & $x_{33}$ & $x_{34}$ & 0 & 0 & 0 \\
\hline 4 & $x_{41}$ & 0 & 0 & $x_{44}$ & $x_{45}$ & 0 & $x_{47}$ \\
\hline 5 & $x_{51}$ & $x_{52}$ & 0 & 0 & $x_{55}$ & $x_{56}$ & 0 \\
\hline 6 & 0 & $x_{62}$ & 0 & $x_{64}$ & 0 & $x_{66}$ & $x_{67}$ \\
\hline
\end{tabular}

Figure 3. The Fano plane $M_{F}$ of Example 4.3 with its non-bases drawn as lines and a circle, and its symbolic slack matrix $S_{M_{F}}(\mathbf{x})$.

EXAMPLE 4.4. Consider the complex matroid $M_{8}$ from [2, p. 33]. It is a rank 3 matroid on 8 elements with non-bases $124,235,346,457,568,167,278$, and 138 . It is depicted with its symbolic slack matrix in Figure 4.

To simplify the computation, we use Corollary 3.2 and note that we can select a representative of each projective equivalence class by fixing certain variables in the slack matrix to be 1 (see Section 5.1 for more details). Fixing the variables $x_{14}, x_{27}$, $x_{28}, x_{3,12}, x_{41}, x_{46}, x_{47}, x_{4,10}, x_{57}, x_{67}, x_{72}, x_{73}, x_{74}, x_{75}, x_{77}, x_{79}, x_{7,11}, x_{7,12}, x_{84}$ to 1 and computing the slack ideal $I_{M_{8}}$ in Macaulay2 [9], we find that it is not the unit ideal. However, it contains the polynomial $x_{8,12}^{2}+x_{8,12}+1$. Since this polynomial has only the complex roots $\frac{-1 \pm i \sqrt{3}}{2}$, we get by Corollary 4.1 that $M_{8}$ is not realizable over $\mathbb{R}$, but it is realizable over $\mathbb{C}$ by Proposition 4.2 .

4.1. Final Polynomials. The method of final polynomials introduced in $[2, \S 4.2]$ certifies when a matroid has no realization. We define an analogous polynomial for the slack ideal, and show how it can be used to improve computational efficiency of checking non-realizability.

Definition 4.5. Let $M$ be a matroid of rank $d+1$. Let $\mathcal{S}$ be the multiplicatively closed set generated by taking finite products of the variables $\mathbf{x}$ in the symbolic slack matrix. A polynomial $f \in \mathbb{k}[\mathbf{x}]$ is a slack final polynomial if

$$
f \in I_{d+2}\left(S_{M}(\mathbf{x})\right) \cap\left(\mathcal{S}+I_{M}\right)
$$

where $I_{d+2}\left(S_{M}(\mathbf{x})\right)$ is the ideal of $(d+2)$-minors of the symbolic slack matrix of $M$.

Intuitively, a slack final polynomial is a $(d+2)$-minor of the symbolic slack matrix that can be written as the sum of a monomial and an element of the slack ideal. We now have the following result, which demonstrates that the existence of slack final polynomials gives a certificate for non-realizability. 


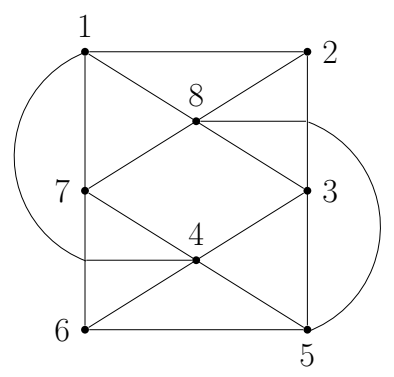

\begin{tabular}{|c|c|c|c|c|c|c|c|c|c|c|c|c|}
\hline \multirow{2}{*}{\multicolumn{2}{|c|}{$\begin{array}{c}H_{1} \\
167\end{array}$}} & $H_{2}$ & $H_{3}$ & $H_{4}$ & $H_{5}$ & $H_{6}$ & $H_{7}$ & $H_{8}$ & $H_{9}$ & $H_{10}$ & $H_{11}$ & $H_{12}$ \\
\hline & & 235 & 568 & 346 & 124 & 278 & 138 & 457 & 48 & 37 & 26 & 15 \\
\hline 1 [ & 0 & $x_{12}$ & $x_{13}$ & $x_{14}$ & 0 & $x_{16}$ & 0 & $x_{18}$ & $x_{19}$ & $x_{1,10}$ & $x_{1,11}$ & 0 \\
\hline 2 & $x_{21}$ & 0 & $x_{23}$ & $x_{24}$ & 0 & 0 & $x_{27}$ & $x_{28}$ & $x_{29}$ & $x_{2,10}$ & 0 & $x_{2,12}$ \\
\hline & $x_{31}$ & 0 & $x_{33}$ & 0 & $x_{35}$ & $x_{36}$ & 0 & $x_{38}$ & $x_{39}$ & 0 & $x_{3,11}$ & $x_{3,12}$ \\
\hline 4 & $x_{41}$ & $x_{42}$ & $x_{44}$ & 0 & 0 & $x_{46}$ & $x_{47}$ & 0 & 0 & $x_{4,10}$ & $x_{4,11}$ & $x_{4,12}$ \\
\hline 5 & $x_{51}$ & 0 & 0 & $x_{54}$ & $x_{55}$ & $x_{56}$ & $x_{57}$ & 0 & $x_{59}$ & $x_{5,10}$ & $x_{5,11}$ & 0 \\
\hline & 0 & $x_{62}$ & 0 & 0 & $x_{65}$ & $x_{66}$ & $x_{67}$ & $x_{68}$ & $x_{69}$ & $x_{6,10}$ & 0 & $x_{6,12}$ \\
\hline & 0 & $x_{72}$ & $x_{73}$ & $x_{74}$ & $x_{75}$ & 0 & $x_{77}$ & 0 & $x_{79}$ & 0 & $x_{7,11}$ & $x_{7,12}$ \\
\hline & $x_{81}$ & $x_{82}$ & 0 & $x_{84}$ & $x_{85}$ & 0 & 0 & $x_{88}$ & 0 & $x_{8,10}$ & $x_{8,11}$ & $\left.x_{8,12}\right\rfloor$ \\
\hline
\end{tabular}

Figure 4. The complex matroid $M_{8}$ of Example 4.4 and its symbolic slack matrix $S_{M_{8}}(\mathbf{x})$.

Proposition 4.6. Let $M$ be a matroid of rank $d+1$. The following are equivalent.

(i) $1 \in I_{M} \subseteq \mathbb{k}[\mathbf{x}]$,

(ii) There is a monomial $m \in \mathbb{k}[\mathbf{x}]$ such that $m \in I_{d+2}\left(S_{M}(\mathbf{x})\right)$,

(iii) A slack final polynomial $f \in I_{d+2}\left(S_{M}(\mathbf{x})\right) \cap\left(\mathcal{S}+I_{M}\right)$ exists for $M$.

REMARK 4.7. Over an algebraically closed field, these conditions are equivalent to the matroid being non-realizable by Proposition 4.2 . When $\mathbb{k}$ is not algebraically closed, these conditions imply non-realizability, but if a matroid is not realizable there may not be a slack final polynomial.

EXAMPLE 4.8. Recall that the complex matroid $M_{8}$ has a complex realization, as $1 \notin I_{M_{8}} \subseteq \mathbb{Q}[\mathbf{x}]$. However, by the above proposition, this means that even though $M_{8}$ is not realizable over $\mathbb{Q}$, it does not have a slack final polynomial.

Proof.

(i) $\Rightarrow$ (iii) Suppose $1 \in I_{M}$. Since $I_{M}$ is the saturation of $I_{d+2}\left(S_{M}(\mathbf{x})\right)$, this implies that there exists a monomial $m \in \mathbb{k}[\mathbf{x}]$ such that $m \cdot 1 \in I_{d+2}\left(S_{M}(\mathbf{x})\right)$. Then, we observe the $m$ is already a slack final polynomial for $M$.

(ii) $\Rightarrow$ (i) If there is a monomial $m \in \mathbb{k}[\mathbf{x}]$ such that $m \in I_{d+2}\left(S_{M}(\mathbf{x})\right)$, then after saturation we find $1 \in I_{M}$.

(iii) $\Rightarrow$ (ii) Suppose $f$ is a slack final polynomial for $M$. Since $f \in\left(\mathcal{S}+I_{M}\right)$, there exists a monomial $m$ and a $g \in I_{M}$ with $f=m+g$. Since $g \in I_{M}$, there exists a monomial $n$ such that $n g \in I_{d+2}\left(S_{M}(\mathbf{x})\right)$, so $n m=n f-n g \in$ $I_{d+2}\left(S_{M}(\mathbf{x})\right)$ is a monomial in $I_{d+2}\left(S_{M}(\mathbf{x})\right)$.

REMARK 4.9. In practice saturation of the ideal $I_{d+2}\left(S_{M}(\mathbf{x})\right)$ can be quite slow, which often makes testing realizability via checking $1 \in I_{M}$ infeasible. Thus the real power of Proposition 4.6 is that one often finds relatively small monomials which are already 
contained in $I_{d+2}\left(S_{M}(\mathbf{x})\right)$. So, if one simply wants to certify non-realizability, a faster method is to compute $I_{d+2}\left(S_{M}(\mathbf{x})\right)$ and check, for example, if $\prod x_{i j} \in I_{d+2}\left(S_{M}(\mathbf{x})\right)$. In the following example we exhibit how this method can be useful for certifying non-realizability.

ExAmple 4.10. Consider the Fano matroid $M_{F}$ of Example 4.3. If we compute $I_{4}\left(S_{M_{F}}(\mathbf{x})\right)$, then we can verify that the product of all of the variables is contained in this ideal. In fact, even the monomial $x_{07} x_{16} x_{25} x_{33} x_{41} x_{52} x_{64}$ is contained in $I_{4}\left(S_{M_{F}}(\mathbf{x})\right)$. Verifying this containment (using the laptop of one of the authors) in Macaulay2 took 0.000067 seconds, while testing $1 \in I_{M_{F}}$ took 3.40765 seconds, indicating a speed up by a factor of 50,000.

ExAmple 4.11. Consider the Vámos matroid pictured in Figure 5. It is a rank 4 matroid $M_{v}$ on 8 elements whose non-bases are given by the sets $1234,1256,3456$, 3478 , and 5678. It is one of the smallest matroids known to be non-realizable over every field. However, the Vámos matroid has 41 hyperplanes, so that its slack matrix is an $8 \times 41$ matrix containing 200 distinct variables. Even computing the full set of minors of this matrix is computationally impractical.

We note though, that it always suffices to show that Proposition 4.6 (ii) holds for some subideal of the ideal of $(d+2)$-minors. In particular, we can look at the minors of a submatrix of $S_{M_{v}}(\mathbf{x})$. Consider the submatrix of the Vámos symbolic slack matrix in Figure 5. One can easily check with Macaulay2 that the monomial given by the product of all the variables in this submatrix is already in the minor ideal of this submatrix (over $\mathbb{Q}$ and various finite fields), making $M_{v}$ non-realizable over these fields by Propositions 4.2 and 4.6.

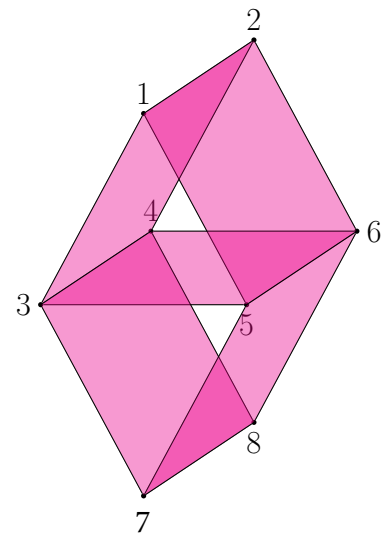

\begin{tabular}{|c|c|c|c|c|c|c|c|c|}
\hline & $H_{1}$ & $H_{2}$ & $H_{3}$ & $H_{4}$ & $H_{5}$ & $H_{6}$ & $H_{7}$ & $H_{8}$ \\
\hline & 3456 & 5678 & 1234 & 3478 & 1256 & 467 & 267 & 127 \\
\hline L & {$\left[x_{11}\right.$} & $x_{12}$ & 0 & $x_{14}$ & 0 & $x_{16}$ & $x_{17}$ & 0 \\
\hline & $x_{21}$ & $x_{22}$ & 0 & $x_{24}$ & 0 & $x_{26}$ & 0 & 0 \\
\hline & 0 & $x_{32}$ & 0 & 0 & $x_{35}$ & $x_{36}$ & $x_{37}$ & $x_{38}$ \\
\hline & 0 & $x_{42}$ & 0 & 0 & $x_{45}$ & 0 & $x_{47}$ & $x_{48}$ \\
\hline & 0 & 0 & $x_{53}$ & $x_{54}$ & 0 & $x_{56}$ & $x_{57}$ & $x_{58}$ \\
\hline & 0 & 0 & $x_{63}$ & $x_{64}$ & 0 & 0 & 0 & $x_{68}$ \\
\hline & $x_{71}$ & 0 & $x_{73}$ & 0 & $x_{75}$ & 0 & 0 & 0 \\
\hline & $x_{81}$ & 0 & $x_{83}$ & 0 & $x_{85}$ & $x_{86}$ & $x_{87}$ & $x_{88}$ \\
\hline
\end{tabular}

Figure 5. The Vámos matroid $M_{v}$ of Example 4.11 with non-bases pictured as planes, and a submatrix of its symbolic slack matrix.

\section{Projective uniqueness of matroids}

The simplest slack realization spaces are those belonging to projectively unique matroids. In this case, we know that there is a single realization up to projective transformations; in other words, $\mathcal{V}\left(I_{M}\right)$ is the toric variety which is the closure of the orbit of some realization under the action of $T_{n, h}$. This implies $\sqrt{I_{M}}=\mathcal{I}\left(\mathcal{V}\left(I_{M}\right)\right)$ is a toric ideal; however, universality suggests that $I_{M}$ need not be radical. A natural question which arises is whether projectively unique matroids correspond exactly to matroids with toric slack ideals. To study this question we introduce an intermediate toric ideal associated to a matroid. 
Definition 5.1. Define the non-incidence graph of matroid $M$ as the bipartite graph $G_{M}$ with one node for each element of the ground set of $M$, one node for each hyperplane, and an edge between element $i$ and hyperplane $H_{j}$ if and only if $i \notin H_{j}$. Notice that $G_{M}$ records the support of the slack matrix $S_{M}$, and so we can think of its edges as being labelled by the corresponding entry of $S_{M}(\mathbf{x})$. (See Figure 6 for an example of the graph $G_{M_{4}}$ for the matroid $M_{4}$ of Example 2.9, and Figure 7 for the non-incidence graph of the non-Fano matroid.)

Let $\mathcal{A}_{G}$ be the set of vectors forming the columns of the vertex-edge incidence matrix of the graph $G$, and let $T_{G}$ be the toric ideal of the vector configuration $\mathcal{A}_{G}$. The toric ideal of a bipartite graph is a well-studied object $[11,14]$. If a matroid $M$ has a slack matrix $S_{M}$ which is a 0-1 matrix, then the toric ideal $T_{G_{M}}$ associated to the graph $G_{M}$ is the ideal of the orbit of $S_{M}$ under the action of the torus $T_{n, h}$. So, $T_{G_{M}}$ describes one projective equivalence class of slack matrices of $M$. We now define an analogous toric ideal for any projective equivalence class.

DeFinition 5.2. Let $M$ be an abstract matroid with realization $V$. Let $\mathbf{s} \in\left(\mathbb{k}^{*}\right)^{t}$ be such that $S_{M[V]}=S_{M}(\mathbf{s})$, where $t$ is the number of variables in $S_{M}(\mathbf{x})$, the symbolic slack matrix of $M$. We define the cycle ideal $C_{V}$ of $M[V]$ to be the ideal

$$
C_{V}=\left\langle\mathbf{x}^{c+}-\alpha_{c} \mathbf{x}^{c-}: \text { c is a cycle in } G_{M} \text { and } \alpha_{c}=\frac{\mathbf{s}^{c+}}{\mathbf{s}^{c-}}\right\rangle \subseteq \mathbb{k}[\mathbf{x}]
$$

where $c+$ and $c$ - are alternating edges from the cycle $c$.

THEOREM 5.3. Let $M$ be a matroid of rank $d+1$ on $n$ elements with $h$ hyperplanes. Let $V$ be a realization of $M$ with slack matrix $S_{M[V]}=\left[s_{i, j}\right]_{i=1, j=1}^{n, h}$. Then the ideal $C_{V}$ is the (scaled) toric ideal which is the kernel of the $\mathbb{k}$-algebra homomorphism $\phi: \mathbb{k}[\mathbf{x}] \rightarrow \mathbb{k}\left[\mathbf{r}, \mathbf{t}, \mathbf{r}^{-1}, \mathbf{t}^{-1}\right]$, which sends $x_{i j} \mapsto s_{i, j} r_{i} t_{j}$.

We note that the cycle ideal of a realization provides a way to distinguish projective equivalence classes of realizations of $M$, as well as detect projective uniqueness.

LEMma 5.4. Let $M$ be a matroid with realizations $U$ and $V$. Then $U, V$ are projectively equivalent if and only if $C_{V}=C_{U}$.

Proof. First suppose that $U$ and $V$ are projectively equivalent. Let $S_{M[V]}$ have entries $s_{i, j}$ for elements $i$ of the ground set of $M$ and hyperplanes $H_{j}$ of $M$. By Lemma 2.6 we know that $S_{M[U]}$ is obtained from $S_{M[V]}$ by scaling the rows by $r_{1}, \ldots, r_{n} \in \mathbb{k}^{*}$ and scaling the columns by $t_{1}, \ldots, t_{h} \in \mathbb{k}^{*}$. Then the entries of $S_{M[U]}$ are $r_{i} t_{j} s_{i, j}$. Since $S_{M[U]}$ and $S_{M[V]}$ have the same support, they have the same cycles. One may then show via elementary calculation that the coefficients $\alpha_{c}$ are the same when calculated from $S_{M[U]}$ and from $S_{M[V]}$ for every cycle $c \in G_{M}$.

Conversely, suppose $C_{V}=C_{U}$. Then $\mathcal{V}\left(C_{V}\right)=\mathcal{V}\left(C_{U}\right)$, and in particular, $S_{M[V]}=$ $S_{M}(\mathbf{s})$ and $S_{M[U]}=S_{M}(\mathbf{u})$ for $\mathbf{s}, \mathbf{u} \in \mathcal{V}\left(C_{V}\right) \cap\left(\mathbb{k}^{*}\right)^{t}$. We now argue that $S_{M[U]}$ can be row and column scaled to be equal to $S_{M[V]}$, which proves the results by Lemma 2.6. Fix a spanning forest $T$ of $G_{M}$. By Lemma 5.11 we may scale the entries in $S_{M}(\mathbf{u})$ corresponding to edges in $T$ to be equal to the corresponding entries of $S_{M[V]}$. Any remaining entry $a$ of $S_{M}(\mathbf{u})$ will correspond to an edge $e$ in $G_{M}$ such that $T \cup\{e\}$ contains a unique cycle $c$, where $e \in c$. The equation $\mathbf{x}^{c+}-\alpha_{c} \mathbf{x}^{c-} \in C_{V}$ corresponding to this cycle must be satisfied by the scaling of $S_{M}(\mathbf{u})$, since $C_{V}=C_{U}$. Since all variables in the cycle except the one labelled by edge $e$ have been fixed, we find that there is only one possible value for $a$. Furthermore, this value equals the corresponding entry in $S_{M[V]}$, since $S_{M[V]}$ satisfies the equations of $C_{V}$ by definition.

COROLlary 5.5. Let $M$ be a matroid with realization $V$. Then, $\mathcal{V}\left(C_{V}\right) \cap\left(\mathbb{k}^{*}\right)^{t}$ consists of exactly the slack matrices of realizations projectively equivalent to $V$. 
LEMMA 5.6. Let $\mathbb{k}$ be algebraically closed and $M$ be a matroid with realization $V$. Then the slack ideal $I_{M}$ is contained in the cycle ideal $C_{V}$.

Proof. By Corollary 5.5 we know that $\mathcal{V}\left(C_{V}\right) \subset \mathcal{V}\left(I_{M}\right)$. Then, $\mathcal{I}\left(\mathcal{V}\left(C_{V}\right)\right) \supset \mathcal{I}\left(\mathcal{V}\left(I_{M}\right)\right)$. Since $C_{V}$ is radical, and since $I_{M} \subset \sqrt{I_{M}}$, this gives $C_{V} \supset I_{M}$.

Proposition 5.7. Let $M$ be a matroid with projectively unique realization $V$. Then $\mathcal{V}\left(I_{M}\right)=\mathcal{V}\left(C_{V}\right)$

Proof. By Corollary 5.5 and Theorem 3.1, we get $\mathcal{V}\left(I_{M}\right) \cap\left(\mathbb{k}^{*}\right)^{t}=\mathcal{V}\left(C_{V}\right) \cap\left(\mathbb{k}^{*}\right)^{t}$. Then since both varieties are irreducible, the result follows.

In fact, we can have $I_{M}=C_{V}$ for a realization $V$ of $M$. In this case, call $I_{M}$ cyclic.

THEOREM 5.8. If the slack ideal of a matroid is cyclic then $M$ is projectively unique and $I_{M}$ is radical. The converse also holds when $\mathbb{k}$ is algebraically closed.

Proof. Suppose that $I_{M}$ is cyclic. Then $M$ is projectively unique by Corollary 5.5 and $I_{M}$ is radical, since it is prime by Theorem 5.3. Conversely, suppose that $M$ is projectively unique and $I_{M}$ is radical. By Proposition 5.7, $\mathcal{I}\left(\mathcal{V}\left(I_{M}\right)\right)=\mathcal{I}\left(\mathcal{V}\left(C_{V}\right)\right.$ ), so that $I_{M}=C_{V}$, as both ideals are radical.

EXAMPLE 5.9. Recall the matroid $M_{4}$ from Example 2.9, whose non-incidence graph $G_{M_{4}}$ is displayed in Figure 6 . The matroid $M_{4}$ has a cyclic slack ideal. Hence $I_{M_{4}}$ is a radical slack ideal, and $M_{4}$ is projectively unique.

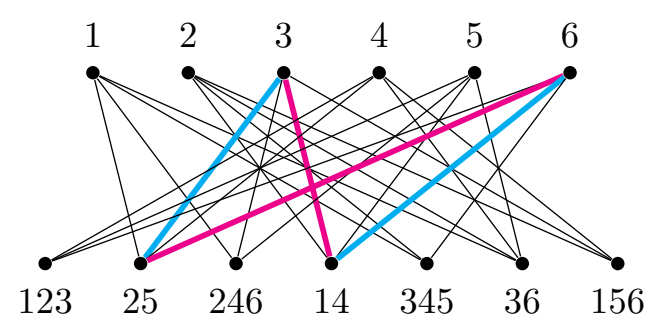

FigurE 6 . The graph $G_{M_{4}}$ for matroid $M_{4}$ with the highlighted cycle corresponding to binomial $x_{36} x_{65}+x_{35} x_{66}$ of Table 1 .

ExAmPLE 5.10. Recall the Fano plane discussed in Example 4.3. Over $\mathbb{F}_{2}$, the 126 binomial generators of $I_{M_{F}}$ found in Example 4.3 correspond to each of the cycles in the graph $G_{M_{F}}$. Over $\mathbb{F}_{2}$ this is the projectively unique representation of $M_{F}$, and this ideal is equal to the cycle ideal of the representation.

5.1. Scaled Slack Matrices. From Corollary 3.2 we know that quotienting by the action of $T_{n, h}$ on $\mathcal{V}\left(I_{M}\right) \cap\left(\mathbb{k}^{*}\right)^{t}$ gives us a realization space for projective equivalence classes of representations of $M$. We now give an explicit way of computing the variety of these equivalence classes. As in [6, Lemma 5.5], we scale rows and columns of a slack matrix via the following lemma, to fix one representative of each projective equivalence class.

LEMMA 5.11. Given a realization of a matroid $M$, we may scale the rows and columns of its slack matrix $S_{M}$ so that it has ones in the entries indexed by the edges in a maximal spanning forest $F$ of the graph $G_{M}$; the resulting realization of $M$ is projectively equivalent to the original realization of $M$. 
DeFinition 5.12. Given a matroid $M$ we can take a symbolic slack matrix and set variables corresponding to edges in a maximal spanning forest $F$ to 1 as in Lemma 5.11 to obtain a scaled symbolic slack matrix. Then, the scaled slack ideal is obtained by taking the $(d+2)$-minors of this matrix and saturating with respect to the product of all the variables.

Using the scaled symbolic slack matrix not only allows us to study the projective realization space of $M$, but also proves to be a useful tool for computations because this matrix will have considerably fewer variables.

EXAmple 5.13. Let $M_{N F}$ be the non-Fano matroid. It is a rank 3 matroid on 7 elements depicted in Figure 7 with its symbolic slack matrix. It differs from the Fano plane by the inclusion of 135 as a basis.

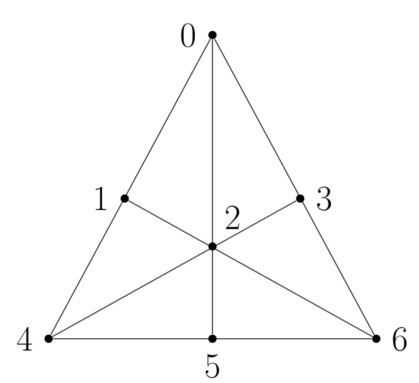

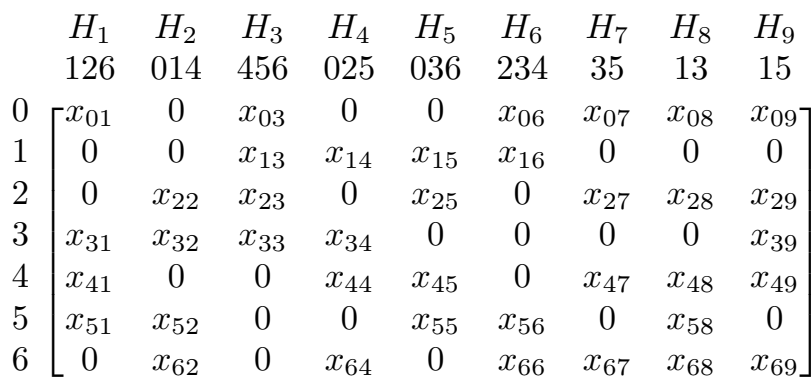

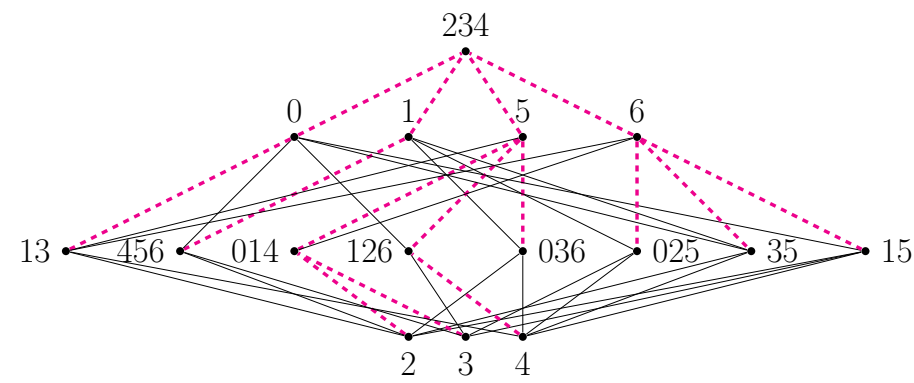

Figure 7 . The non-Fano matroid $M_{N F}$, with its non-bases depicted as lines, together with its symbolic slack matrix $S_{M_{N F}}(\mathbf{x})$ and the spanning tree $F$ selected in Example 5.13.

We now show that the non-Fano matroid is projectively unique, and write down a realization from a slack matrix. Let $F$ be the spanning tree of $G_{M_{N F}}$ depicted with dotted lines in Figure 7 . We set the corresponding variables $x_{41}, x_{51}, x_{22}, x_{32}, x_{52}$, $x_{13}, x_{64}, x_{55}, x_{06}, x_{16}, x_{56}, x_{66}, x_{67}, x_{08}, x_{69}$ to 1 in the symbolic slack matrix in Figure 7. Taking the ideal of 4-minors and saturating, we find that the ideal consists of equations of the form $x_{i j}-\alpha_{i j}$, for $\alpha_{i j} \in \mathbb{Q}$, so the configuration is projectively unique over $\mathbb{Q}$. The slack matrix corresponding to the single point in $\mathcal{V}\left(I_{M}\right) \cap\left(\mathbb{k}^{*}\right)^{39} / T_{7,9}$ is

$$
\left(\begin{array}{ccccccccc}
1 & 0 & 1 & 0 & 0 & 1 & 1 & 1 & 1 \\
0 & 0 & 1 & 1 & -1 & 1 & 2 & 0 & 0 \\
0 & 1 & -1 & 0 & 1 & 0 & -1 & -1 & 1 \\
-1 & 1 & -1 & 1 & 0 & 0 & 0 & -2 & 0 \\
1 & 0 & 0 & -1 & 1 & 0 & -1 & 1 & 1 \\
1 & 1 & 0 & 0 & 1 & 1 & 0 & 0 & 2 \\
0 & 1 & 0 & 1 & 0 & 1 & 1 & -1 & 1
\end{array}\right)
$$


ExAmple 5.14. Consider the Perles configuration $M_{\star}$ of Figure 8. It is a rank 3 matroid on 9 elements with hyperplanes given by $0678,347,156,128,045,358,013$, 246, 257, 48, 17, 36, 14, 23, 02. Its symbolic slack matrix is shown in Figure 8 and has the matrix $S(\mathbf{x})$ studied in $[6, \S 4.3]$ as a submatrix.

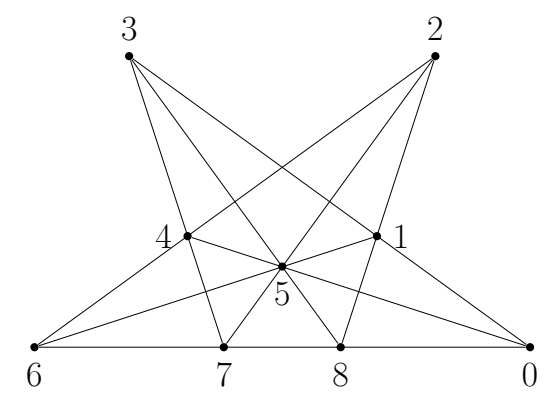

\begin{tabular}{|c|c|c|c|c|c|c|c|c|c|c|c|c|c|c|c|}
\hline & $H_{1}$ & $H_{2}$ & $H_{3}$ & $H_{4}$ & $H_{5}$ & $H_{6}$ & $H_{7}$ & $H_{8}$ & $H_{9}$ & $H_{10}$ & $H_{11}$ & $H_{12}$ & $H_{13}$ & $H_{14}$ & $H_{15}$ \\
\hline & 678 & 347 & 156 & 128 & 045 & 358 & 013 & 246 & 257 & 48 & 17 & 36 & 14 & 23 & 02 \\
\hline 0 & {$[0$} & $x_{02}$ & $x_{03}$ & $x_{04}$ & 0 & $x_{06}$ & 0 & $x_{08}$ & $x_{09}$ & $x_{0,10}$ & $x_{0,11}$ & $x_{0,12}$ & $x_{0,13}$ & $x_{0,14}$ & 0 \\
\hline 1 & $x_{11}$ & $x_{12}$ & 0 & 0 & $x_{15}$ & $x_{16}$ & 0 & $x_{18}$ & $x_{19}$ & $x_{1,10}$ & 0 & $x_{1,12}$ & 0 & $x_{1,14}$ & $x_{1,15}$ \\
\hline 2 & $x_{21}$ & $x_{22}$ & $x_{23}$ & 0 & $x_{25}$ & $x_{26}$ & $x_{27}$ & 0 & 0 & $x_{2,10}$ & $x_{2,11}$ & $x_{2,12}$ & $x_{2,13}$ & 0 & 0 \\
\hline 3 & $x_{31}$ & 0 & $x_{33}$ & $x_{34}$ & $x_{35}$ & 0 & 0 & $x_{38}$ & $x_{39}$ & $x_{3,10}$ & $x_{3,11}$ & 0 & $x_{3,13}$ & 0 & $x_{3,15}$ \\
\hline 4 & $x_{41}$ & 0 & $x_{43}$ & $x_{44}$ & 0 & $x_{46}$ & $x_{47}$ & 0 & $x_{49}$ & 0 & $x_{4,11}$ & $x_{4,12}$ & 0 & $x_{4,14}$ & $x_{4,15}$ \\
\hline 5 & $x_{51}$ & $x_{52}$ & 0 & $x_{54}$ & 0 & 0 & $x_{57}$ & $x_{58}$ & 0 & $x_{5,10}$ & $x_{5,11}$ & $x_{5,12}$ & $x_{5,13}$ & $x_{5,14}$ & $x_{5,15}$ \\
\hline 6 & 0 & $x_{62}$ & 0 & $x_{64}$ & $x_{65}$ & $x_{66}$ & $x_{67}$ & 0 & $x_{69}$ & $x_{6,10}$ & $x_{6,11}$ & 0 & $x_{6,13}$ & $x_{6,14}$ & $x_{6,15}$ \\
\hline 7 & 0 & 0 & $x_{73}$ & $x_{74}$ & $x_{75}$ & $x_{76}$ & $x_{77}$ & $x_{78}$ & 0 & $x_{7,10}$ & 0 & $x_{7,12}$ & $x_{7,13}$ & $x_{7,14}$ & $x_{7,15}$ \\
\hline 8 & 0 & $x_{82}$ & $x_{83}$ & 0 & $x_{85}$ & 0 & $x_{87}$ & $x_{88}$ & $x_{89}$ & 0 & $x_{8,11}$ & $x_{8,12}$ & $x_{8,13}$ & $x_{8,14}$ & $x_{8,15}$ \\
\hline
\end{tabular}

Figure 8. The Perles configuration matroid $M_{\star}$ of Example 5.14 with non-bases shown as lines, and its symbolic slack matrix $S_{M_{*}}(\mathbf{x})$.

Since the ideal of $S(\mathbf{x})$ will be contained in the ideal of the whole matrix $S_{M_{\star}}(\mathbf{x})$, it follows from computation in [6] that $M_{\star}$ is not realizable over $\mathbb{Q}$. However, it is realizable over $\mathbb{R}$, and computing its scaled slack ideal we find that the slack variety consists of the following matrices, where $\alpha$ is a root of the polynomial $\alpha^{2}-3 \alpha+1$ :

$$
\left[\begin{array}{ccccccccccccccc}
0 & 1 & \alpha-3 & 3-\alpha & 0 & 3-\alpha & 0 & -1 & 1 & 3-\alpha & 1 & \alpha-2 & 2-\alpha & 2-\alpha & 0 \\
2 \alpha-5 & 1 & 0 & 0 & 3-\alpha & 3-\alpha & 0 & 2-\alpha & 3-\alpha & \alpha-2 & 0 & \alpha-2 & 0 & 2-\alpha & 5-2 \alpha \\
1 & \alpha & 1 & 0 & \alpha & \alpha-1 & 1 & 0 & 0 & \alpha & 1-\alpha & 1 & \alpha & 0 & 0 \\
1 & 0 & \alpha-1 & 1-\alpha & \alpha-1 & 0 & 0 & \alpha-1 & -\alpha & 1 & 1-2 \alpha & 0 & \alpha & 0 & -1 \\
2-\alpha & 0 & 2-\alpha & \alpha-1 & 0 & \alpha-2 & 1 & 0 & \alpha-1 & 0 & \alpha & 2-\alpha & 0 & \alpha-1 & \alpha-1 \\
2-\alpha & 1-\alpha & 0 & 1 & 0 & 0 & 1 & \alpha-1 & 0 & 2-\alpha & 1 & 1-\alpha & 1 & \alpha & \alpha-1 \\
0 & 1 & 0 & 1 & 1 & 1 & 1 & 0 & 1 & 1 & 1 & 0 & 1 & 1 & 1 \\
0 & 0 & 3-\alpha & \alpha-2 & 1 & \alpha-2 & 1 & 1 & 0 & \alpha-2 & 0 & 2-\alpha & \alpha-1 & \alpha-1 & 1 \\
0 & \alpha+1 & 1 & 0 & 1 & 0 & 1 & \alpha & 1-\alpha & 0 & 1-\alpha & 1-\alpha & \alpha & \alpha & 1
\end{array}\right]
$$

Over any field where 5 has a square root, the variety has degree 2 and dimension 0 , so it consists of two points obtained by setting $\alpha=\frac{3 \pm \sqrt{5}}{2}$. 


\section{Appendix A. Notation}

\begin{tabular}{|ll|}
\hline Name & Description \\
\hline$\alpha_{H}$ & a hyperplane normal to the hyperplane $H$ \\
$C_{V}$ & cycle ideal of realization $V$ \\
$G_{M}$ & non-incidence graph of $M$ \\
$I_{M}$ & slack ideal of $M$, see Definition 2.8 \\
$M[V]$ & matroid given by realization $V$ \\
$M_{H}$ & Plücker substitution matrices, see Definition 3.7 \\
$S_{M}$ & slack matrix of $M$, see Definition 2.1 \\
$S_{M}(\mathbf{x})$ & symbolic slack matrix, see Definition 2.8 \\
$t$ & number of slack variables \\
$T_{n, h}$ & torus of row and column scalings $\left(\mathbb{k}^{*}\right)^{n} \times\left(\mathbb{k}^{*}\right)^{h}$ \\
$U_{M}$ & the universal realization ideal of $M$, see Definition 3.7 \\
$\mathcal{V}\left(I_{M}\right)$ & slack variety of $M$, see Definition 2.8 \\
$x_{i j}$ & slack variables, see Definition 2.8 \\
\hline
\end{tabular}

Acknowledgements. We would like to thank Bernd Sturmfels and Rekha Thomas for their guidance and helpful discussion. We also thank Dan Corey for pointing us to examples of matroids with interesting realization spaces.

The software package Macaulay2 [9] was invaluable in calculating all of the examples from this paper. In addition, the package "Matroids" written for Macaulay2 by Justin Chen was indispensable.

We also acknowledge the Mathematical Sciences Research Institute, the MaxPlanck-Institut für Mathematik in den Naturwissenschaften, and the University of Washington for facilitating our collaboration on this paper.

\section{REFERENCES}

[1] A. Björner, M. Las Vergnas, B. Sturmfels, N. White, and G. Ziegler, Oriented matroids, Cambridge University Press, 1993.

[2] J. Bokowski and B. Sturmfels, Computational synthetic geometry, Lecture Notes in Mathematics, vol. 1355, Springer-Verlag, Berlin, 1989.

[3] J. Chen, Matroids: A macaulay2 package, https://arxiv.org/abs/1511.04618, 2015.

[4] D. A. Cox, J. Little, and D. O'Shea, Ideals, varieties, and algorithms. An introduction to computational algebraic geometry and commutative algebra, fourth ed., Undergraduate Texts in Mathematics, Springer, Cham, 2015.

[5] G. Gordon and J. McNulty, Matroids: a geometric introduction, Cambridge University Press, Cambridge, 2012.

[6] J. Gouveia, A. Macchia, R. R. Thomas, and A. Wiebe, The slack realization space of a polytope, https://arxiv.org/abs/1708.04739.

[7] J. Gouveia, P. A. Parrilo, and R. R. Thomas, Lifts of convex sets and cone factorizations, Math. Oper. Res. 38 (2013), no. 2, 248-264.

[8] J. Gouveia, K. Pashkovich, R. Z. Robinson, and R. R. Thomas, Four-dimensional polytopes of minimum positive semidefinite rank, J. Combin. Theory Ser. A 145 (2017), 184-226.

[9] D. R. Grayson and M. E. Stillman, Macaulay2, a software system for research in algebraic geometry, Available at http://www.math.uiuc.edu/Macaulay2/.

[10] N. E. Mnëv, The universality theorems on the classification problem of configuration varieties and convex polytopes varieties, in Topology and geometry - Rohlin Seminar, Lecture Notes in Math., vol. 1346, Springer, Berlin, 1988, pp. 527-543.

[11] H. Ohsugi and T. Hibi, Toric ideals generated by quadratic binomials, J. Algebra 218 (1999), no. $2,509-527$.

[12] J. Oxley, Matroid theory, second ed., Oxford Graduate Texts in Mathematics, vol. 21, Oxford University Press, Oxford, 2011. 
[13] T. Rothvoss, The matching polytope has exponential extension complexity, in STOC'14 - Proceedings of the 2014 ACM Symposium on Theory of Computing, ACM, New York, 2014, pp. 263272.

[14] R. H. Villarreal, Rees algebras of edge ideals, Comm. Algebra 23 (1995), no. 9, 3513-3524.

[15] M. Yannakakis, Expressing combinatorial optimization problems by linear programs, J. Comput. System Sci. 43 (1991), no. 3, 441-466.

Madeline Brandt, University of California, Berkley, Dept. of mathematics, 970 Evans Hall, Berkeley, CA 94720, USA

E-mail : brandtm@berkeley.edu

Amy Wiebe, University of Washington, Dept. of mathematics, Box 354350, Seattle, WA 98195, USA

E-mail : awiebe@uw.edu 